\title{
Effect of the non-specific matrix metalloproteinase inhibitor Doxycycline on endometriotic implants in an experimental rat model
}

\author{
UMIT GOKTOLGA $^{1}$, SABRI CAVKAYTAR ${ }^{2}$, SADIMAN KIYKAC ALTINBAS ${ }^{1}$, \\ OMER LUTFI TAPISIZ ${ }^{1}$, ANIL TAPISIZ ${ }^{3}$ and OZLEM ERDEM ${ }^{4}$
}

${ }^{1}$ Department of Obstetrics and Gynecology, Etlik Zubeyde Hanim Women's Health Training and Research Hospital;
${ }^{2}$ Department of Obstetrics and Gynecology, Zekai Tahir Burak Women's Health Education and Research Hospital;
Departments of ${ }^{3}$ Pediatric Infectious Diseases and ${ }^{4}$ Pathology, Gazi University Faculty of Medicine, Ankara, Turkey

Received May 31, 2014; Accepted January 20, 2015

DOI: $10.3892 /$ etm.2015.2304

\begin{abstract}
The aim of this study was to investigate the possible therapeutic effects of Doxycycline (Dox) on endometriotic lesions in an experimental rat model. Thirty-seven female Wistar albino rats with surgically induced endometriosis were randomized and divided into four groups. The rats were administered $5 \mathrm{mg} / \mathrm{kg} /$ day oral Dox in Group 1 (low-dose Dox group, $\mathrm{n}=9$ ), $20 \mathrm{mg} / \mathrm{kg} /$ day oral Dox in Group 2 (high-dose Dox group, $\mathrm{n}=10$ ) and $1 \mathrm{mg} / \mathrm{kg}$ single dose, subcutaneous leuprolide acetate in Group 3 (leuprolide acetate group, $n=9$ ). The rats in Group 4 (control group, $n=9$ ) were given no medication. The rats received medication for three weeks and were then sacrificed to evaluate the morphological and histological features of the implants. Matrix metalloproteinase (MMP)-9 immunoreactivity of the implants was also evaluated. The size of the endometriotic implants decreased in Groups 1-3 but statistically significant differences were not observed among the groups. The mean surface area of the endometriotic implants decreased from $69.3 \pm 30.8$ to $52.1 \pm 27.0 \mathrm{~mm}^{2}$ in Group $1(\mathrm{P}>0.05)$, from $60.2 \pm 18.9$ to $38.6 \pm 28.7 \mathrm{~mm}^{2}$ in Group $2(\mathrm{P}>0.05)$ and from $58.1 \pm 33.1$ to $26 \pm 9.0 \mathrm{~mm}^{2}$ in Group 3 ( $\mathrm{P}=0.03)$. The epithelial MMP-9 immunohistochemical score was significantly higher in Group 1 and lower in Group 3 when compared with the control group (Group 4) $(\mathrm{P}=0.042$ and $\mathrm{P}=0.014$, respectively). When the stromal MMP-9 immunohistochemical and histopathological scores of the endometriotic implants were compared, no statistically significant differences were found among the groups. Although
\end{abstract}

Correspondence to: Dr Omer Lutfi Tapisiz, Department of Obstetrics and Gynecology, Etlik Zubeyde Hanim Women's Health Training and Research Hospital, Yeni Etlik Cad., No. 55, Etlik, Kecioren, Ankara 06010, Turkey

E-mail: omertapisiz@yahoo.com.tr

Key words: Doxycycline, leuprolide acetate, rat, endometriosis, matrix metalloproteinase 9 there was no statistically significant difference, Dox reduced the endometriotic implant area in the rat endometriosis model. Further studies are required to investigate the potential efficacy of Dox in endometriosis due to its widespread use and tolerability.

\section{Introduction}

Endometriosis, defined by the presence of endometrial glands and stroma outside the uterine cavity, is a common cause of pelvic pain and infertility in females of a reproductive age (1). Although endometriosis is clinically well described, the complete mechanism of the disease remains unclear. The Sampson hypothesis, regarding the implantation of endometrial sheddings outside of the uterine cavity via retrograde menstruation, is the most accepted theory (2). It has previously been shown that increased expression of matrix metalloproteinases (MMPs) is necessary for the adherence and invasion of ectopic endometrium in endometriotic lesions (3).

The MMPs, a family of endoproteases, are involved in extracellular matrix and basement membrane degradation, which are key steps for cellular migration and invasion. These endoproteases mediate a large variety of normal biological processes, including embryonic development, ovulation and menstruation. Adversely, their great variability provides a complex intervention in pathological conditions such as arthritis, atherosclerosis, cancer, inflammatory disorders and endometriosis (4). The expression of various MMPs has been demonstrated in endometriosis $(5,6)$. In experimentally induced endometriosis models, the prevention of endometriotic lesions has been achieved by MMP inhibitors (7) or the downregulation of MMP expression (8).

Doxycycline (Dox) is a second-generation, lipid-soluble tetracycline derivative that has numerous cellular effects on proliferation, migration, apoptosis and matrix remodeling (9). Independently of antimicrobial activity, Dox and other tetracycline derivatives are non-specific MMP inhibitors $(10,11)$. Furthermore, Dox has various non-antibiotic properties, including the blockage of apoptosis, angiogenesis and the 
inflammatory process (12). Based on this evidence, the aim of the present study was to investigate the possible therapeutic potential of Dox as a non-specific MMP inhibitor in a rat endometriosis model.

\section{Materials and methods}

Animals. Forty mature, nonpregnant female Wistar albino rats weighing between 200 and $230 \mathrm{~g}$ were provided by the Animal Research Center of the Ankara Education and Research Hospital (Ankara, Turkey). The rats were caged in a controlled environment of $22^{\circ} \mathrm{C}$ with 12 -h light/dark cycles. All rats were observed for one week to ascertain health prior to surgery. The study was approved by the Institutional Review Board of the Ankara Teaching and Research Hospital (Ankara, Turkey) and carried out at the Animal Research Center of the same hospital. All investigations complied with the 1996 Guide for the Care and Use of Laboratory Animals from the National Academy of Science.

Surgical procedures. The rats were anesthetized by intramuscular administration of $4 \mathrm{mg} / \mathrm{kg}$ ketamine hydrochloride and $0.04 \mathrm{mg} / \mathrm{kg}$ xylazine hydrochloric acid. All procedures were carried out under sterile conditions. A 3-4-cm vertical midline incision was made to expose the reproductive organs. Three consecutive laparotomies were performed in each rat.

First surgery: Induction of endometriosis. Endometriosis was induced surgically by transplanting an autologous fragment of uterine tissue onto the inner surface of the abdominal wall, as proposed by Vernon and Wilson (13) with minor modifications by Lebovic et al (14). Following the exposure of the uterine horns by a $3-4-\mathrm{cm}$ vertical midline incision, the right uterine horn was ligated with a 3-0 polyglactin suture and a $1-\mathrm{cm}$ segment was removed. The excised horn was cut and placed in sterile phosphate-buffered saline (PBS) at $37^{\circ} \mathrm{C}$ and divided into $5 \times 5 \mathrm{~mm}$ pieces. One trimmed piece of uterine tissue (endometrium and myometrium) was implanted onto the inner surface of the left abdominal wall with the serosal surface apposed and sutured with a single 3-0 polyglactin suture to the abdominal wall. As a result, the endometrium was in contact with the peritoneal surface. Prior to the closure of the abdominal wall, $2 \mathrm{ml}$ saline was administered into the abdominal cavity to prevent drying and minimize adhesion formation. The midline incision was closed in a continuous interlocking manner with 3-0 polyglactin sutures. Following the first surgery, all rats were observed for 21 days without any medication.

Second surgery: Evaluation of endometriotic foci. Following the first surgery, three rats died. The remaining 37 rats underwent a second exploratory laparotomy to examine the endometrial implants (Fig. 1). The length and width of the implants were measured using a caliper and the surface area $\left[\mathrm{mm} \mathrm{x} \mathrm{mm}\left(\mathrm{mm}^{2}\right)\right]$ of the implants was calculated.

The rats were randomly assigned to one of four groups. Group 1 rats (low-dose Dox group, $n=9$ ) were administered $5 \mathrm{mg} / \mathrm{kg} / \mathrm{day}$ oral Dox (Monodoks tablet; Deva, Istanbul, Turkey). Group 2 rats (high-dose Dox group, $n=10$ ) were administered $20 \mathrm{mg} / \mathrm{kg} /$ day oral Dox. The rats in Group 3
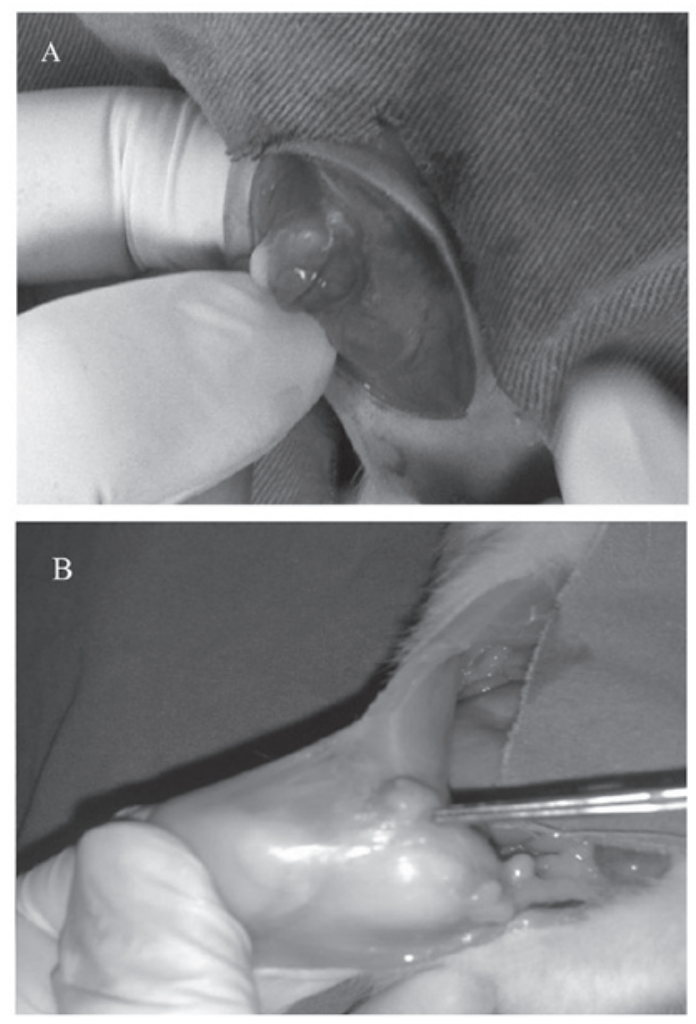

Figure 1. (A) Formation of the endometriotic implant 21 days after the implantation of endometrial tissue. (B) Regression of the endometrial implant in the leuprolide acetate group 21 days after the treatment.

(gonadotrophin-releasing hormone agonist group, $\mathrm{n}=9$ ) were administered a single subcutaneous (s.c.) injection of leuprolide acetate depot formulation $\left(1 \mathrm{mg} / \mathrm{kg}\right.$ body weight; Lucrin ${ }^{\circledR}$; Abbott Laboratories S.A., Istanbul, Turkey). Group 4 rats (control group, $n=9$ ) were given no medication. The selection of Dox $(9,15)$ and leuprolide acetate $(15)$ dosages was based on previous studies. The drugs were administered via an orogastric tube, and all rats were observed for 21 days.

Third surgery: Effects of Dox and leuprolide acetate. Following randomization, while taking the medication, two rats from Group 3 and one rat from Group 4 died due to infection or complications of surgery. A total of 34 rats completed the study. Following a 21-day treatment period, the rats underwent a final laparotomy and the rats were sacrificed using ketamine anesthesia. The length and width of the implants were measured and the surface area of the implants was calculated for the second time. The implants were then excised and fixed in $10 \%$ formalin for histopathological examination. All surgical procedures were performed physicians who were blinded to the groups, and all measurements were performed by the same physician.

Histopathological and immunohistochemical examination. The endometriotic implants were first fixed in $10 \%$ formaldehyde solution and then embedded in paraffin and cut into 4-mm sections. These sections were stained with hematoxylin and eosin and examined under a light microscope. The persistence of epithelial cells in the endometriotic implants was evaluated 
semiquantitatively and scored as follows: 0 , no epithelium; 1 , poorly preserved epithelium; 2 , moderately preserved epithelium with leukocyte infiltrate; 3 , well-preserved epithelial layer (16).

Sections $(4-\mu \mathrm{m})$ from each formalin-fixed, paraffin-embedded endometriotic implant were used for the immunostaining. Following deparaffinization and rehydration, $\mathrm{H}_{2} \mathrm{O}_{2}(3 \%)$ was applied to each section and washed with PBS. The sections were incubated with the primary monoclonal antibody against MMP-9 (1:200; 4A3, cat. no. NBP2-13173; Novus Biologicals LLC, Littleton, CO, USA) overnight at $4^{\circ} \mathrm{C}$ and then washed two times in PBS and incubated with biotinylated immunoglobulin G (ScyTek Laboratories, Inc., Logan, UT, USA) as the secondary antibody for $15 \mathrm{~min}$. Following incubation, the sections were rewashed with PBS two times and incubated with streptavidin-biotin-peroxidase complex (ScyTek Laboratories, Inc.) for $15 \mathrm{~min}$. The specimens were then placed in 3-amino-9-ethylcarbazole chromogen (ScyTek Laboratories, Inc.) for $5 \mathrm{~min}$. Subsequent to counterstaining with hematoxylin, the slides were examined with a light microscope. Immunoreactivity was evaluated by the same pathologist, who was blinded to the study groups. A semi-quantitative histological score (HSCORE) was used and calculated with the following formula: HSCORE $=\Sigma \pi(I+1)$, where ' $\pi$ ' represents the percentage of stained epithelial and stromal cells for each intensity and 'I' represents the staining intensity ( 0 , no labelling; 1 , weak; 2 , moderate; 3 , strong) (16).

Statistical analysis. Data were stored and analyzed using the Statistical Package for the Social Sciences version 13.0 (SPSS, Inc., Chicago, IL, USA). All values are presented as the median (minimum-maximum) and mean \pm standard deviation. Normality was tested by the Shapiro-Wilk test. Non-normally distributed metric variables were analyzed by the Kruskal-Wallis test for comparisons of the variables among the groups. If the difference was significant, the Mann-Whitney U test with Bonferroni correction was used. The mean surface areas of the endometriotic implants in the same group (prior and subsequent to the medical treatment) were analyzed by Wilcoxon's signed-rank test. $\mathrm{P}<0.05$ was considered to indicate a statistically significant difference.

\section{Results}

Study mortality. Following the first laparotomy, three rats died within a few days, most likely due to complications of the surgery. A total of 37 rats with established viable endometriosis were randomized prior to treatment. At the beginning of the medical treatment, the surface area of the endometriotic implants was similar in the four groups $(\mathrm{P}=0.125)$ (Table I). Following randomization, while taking the medication, two rats from Group 3 and one rat from Group 4 died due to infection or complications of surgery. A total of 34 rats completed the study.

Implant area. After the 21-day treatment period, the size of the endometriotic implants decreased in Groups 1-3 but statistically significant differences were not observed among the groups (Table I). The mean surface area of the endometriotic implants decreased from $69.3 \pm 30.8$ to $52.1 \pm 27.0 \mathrm{~mm}^{2}$

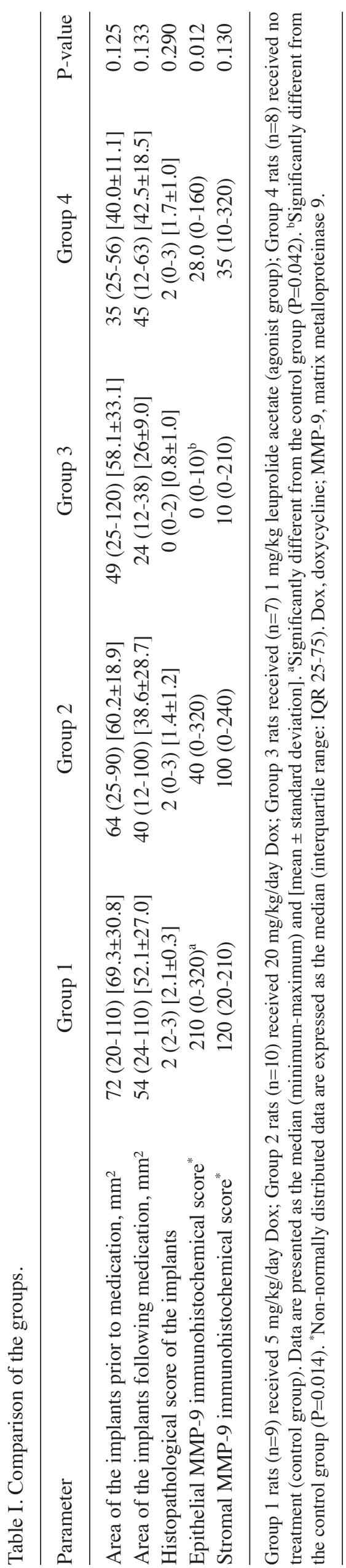


Table II. Changes in the implant area prior and subsequent to treatment.

Area of the implant $\left(\mathrm{mm}^{2}\right)$

\begin{tabular}{lccr}
\cline { 2 - 4 } Group & Prior to treatment & Following treatment & P-value $^{\mathrm{a}}$ \\
\hline 1 & $72(20-110)[69.3 \pm 30.8]$ & $54(24-110)[52.1 \pm 27.0]$ & 0.207 \\
2 & $64(25-90)[60.2 \pm 18.9]$ & $40(12-100)[38.6 \pm 28.7]$ & 0.093 \\
3 & $49(25-120)[58.1 \pm 33.1]$ & $24(12-38)[26.0 \pm 9.0]$ & $\mathbf{0 . 0 3 0}$ \\
4 & $35(25-56)[40.0 \pm 11.1]$ & $45(12-63)[42.5 \pm 18.5]$ & 0.674 \\
P-value $^{\mathrm{b}}$ & 0.125 & 0.133 & \\
\hline
\end{tabular}

Group 1 rats (n=9) received $5 \mathrm{mg} / \mathrm{kg}$ /day Dox; Group 2 rats $(\mathrm{n}=10)$ received $20 \mathrm{mg} / \mathrm{kg} /$ day Dox; Group 3 rats ( $\mathrm{n}=7$ ) received $1 \mathrm{mg} / \mathrm{kg}$ leuprolide acetate (agonist group); Group 4 rats $(\mathrm{n}=8)$ received no treatment (control group). Data are presented as the median (minimum-maximum) and

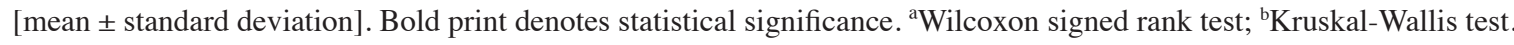
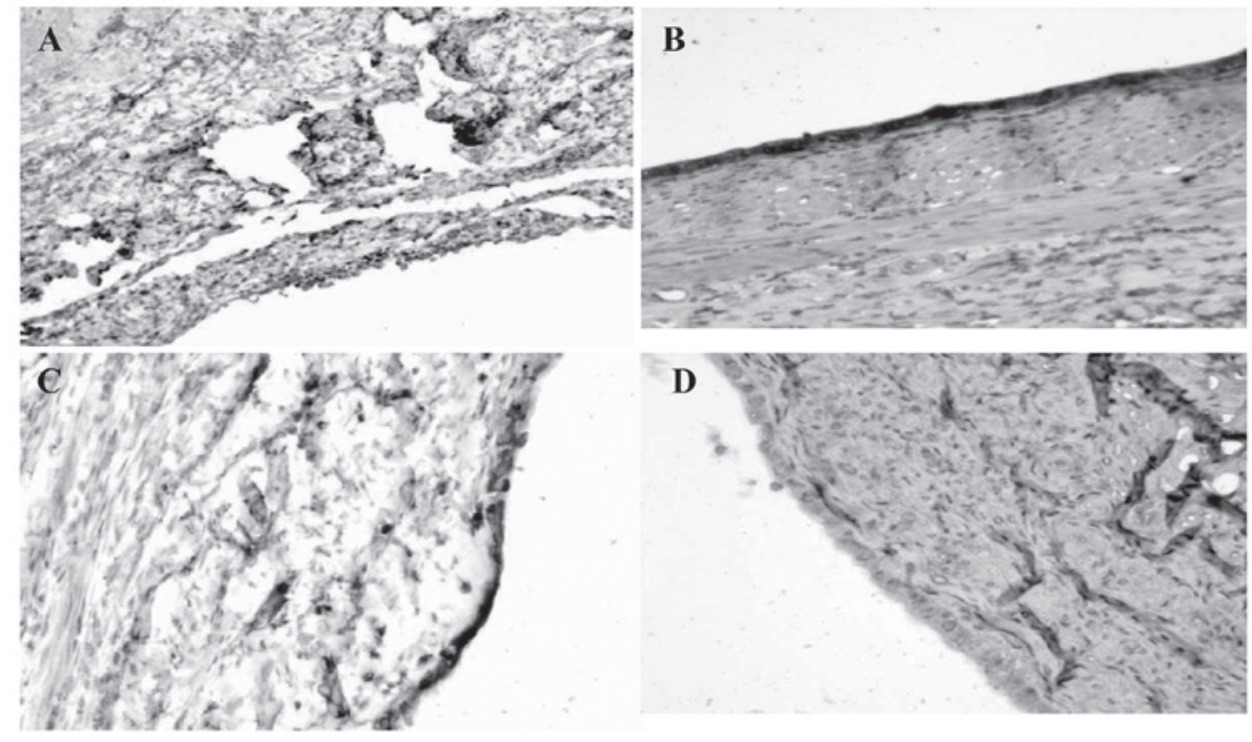

Figure 2. Immunohistochemical MMP-9 staining of the endometriotic implants at the end of the treatment. (A) Leuprolide acetate group (MMP-9 epithelial score, 0; magnification, x10); (B) high-dose Dox group (MMP-9 epithelial score, 320; magnification, x10); (C) low-dose Dox group (MMP-9 epithelial score, 60; magnification, x20); (D) control group (MMP-9 epithelial score, 160; magnification, x20). MMP-9, matrix metalloproteinase 9.

in Group $1(\mathrm{P}>0.05)$, from $60.2 \pm 18.9$ to $38.6 \pm 28.7 \mathrm{~mm}^{2}$ in Group $2(\mathrm{P}>0.05)$ and from $58.1 \pm 33.1$ to $26.0 \pm 9.0 \mathrm{~mm}^{2}$ in Group $3(\mathrm{P}=0.03)$ (Table II). In the control group, the mean surface area of the endometriotic implants did not change significantly $(\mathrm{P}>0.05)$ (Table II).

Histophathological and immunohistochemical scores. The mean histopathological score of the endometriotic implants was lower in Group 3 than that in the other groups, but no statistically significant differences were found $(\mathrm{P}>0.05)$ (Table I). Immunohistochemical staining for MMP-9 in the endometrial implants was performed and evaluated in the stromal and epithelial compartments at the end of treatment (Fig. 2); although the stromal MMP-9 immunohistochemical score was lower in Group 3 than that in the other groups, the difference was not statistically significant $(\mathrm{P}=0.13)$ (Table I). The epithelial MMP-9 immunohistochemical score was significantly higher in Group 1 and lower in Group 3 when compared with the control group (Group 4) ( $\mathrm{P}=0.042$ and $\mathrm{P}=0.014$, respectively) (Fig. 2).

\section{Discussion}

The aim of the present study was to compare the possible therapeutic effects of Dox and leuprolide acetate in a rat endometriosis model. Although the mean area of the endometriotic implants decreased in Groups 1-3 when compared with the control, no statistically significant difference was found among the groups. The MMP-9 immunostaining of the specimens was also examined in order to provide more objective results. No statistically significant difference was found in the mean histopathological score of the endometriotic implants and the stromal MMP-9 immunohistochemical score among the groups; however, the epithelial MMP-9 immunohistochemical score was significantly higher in Groups 1 and lower in group 3 when compared with the control group.

MMPs are a large family of zinc-dependent proteinases that are involved in extracellular matrix degradation and interfere with numerous physiological and pathological processes, 
including development, angiogenesis, ovulation, cancer, atherosclerosis and endometriosis (17). MMP-9 is the most complex family member for the remodeling of the extracellular matrix, and increased MMP-9 expression by eutopic and ectopic endometrial tissue in females with endometriosis has been reported and associated with the severity of endometriosis $(6,18,19)$.

Dox is a broad-spectrum, lipid-soluble tetracycline derivative and has numerous non-antibiotic effects, including inhibition of inflammation, angiogenesis and apoptosis (20). Non-specific inhibition of MMPs by Dox in certain tissues at different doses has been demonstrated $(9,21)$. It has been reported that Dox is a strong inhibitor of MMP-2 and MMP-9 (22,23). Since MMPs are necessary for normal physiological processes, excessive inhibition of MMPs by synthetic MMP inhibitors may result in serious side effects $(24,25)$; therefore, less potent MMP inhibitors, such as Dox, have been found to be safer and effective in certain pathological conditions, such as endometriosis $(15,26)$. The effect of Dox is dose- and cell-dependent. The daily oral dose of Dox in rats varies between 5 and $100 \mathrm{mg} / \mathrm{kg} /$ day in the literature $(15,27)$.

In the only study investigating the effect of Dox in a rat endometriosis model, rats were administered 5 and $40 \mathrm{mg} / \mathrm{kg} / \mathrm{day}$ Dox orally, and low-dose Dox caused an increased regression of the endometriotic implants in rats (15). Akkaya et al (15) reported statistically significant decreases in the size of the endometriotic implants in the leuprolide acetate (1 mg/kg, s.c.), low-dose Dox (5 mg/kg/day) and high-dose Dox (40 mg/kg/day) groups when compared with the control group. With reference to the MMP-2 and MMP-9 immunostaining of the endometriotic implants, the authors concluded that low-dose Dox ( $5 \mathrm{mg} / \mathrm{kg} /$ day) was as effective as leuprolide acetate in stimulating the regression of the endometriotic implants (15). To avoid the toxic effects of Dox and to optimize the Dox dose in the rat endometriosis model, the doses used in the present study were 5 and $20 \mathrm{mg} / \mathrm{kg} / \mathrm{day}$. In this study, the mean area of the endometriotic implants decreased in the leuprolide acetate (1 mg/kg s.c.), low-dose Dox (5 mg/kg/day) and high-dose Dox (20 mg/kg/day) groups; however, no statistically significant difference was found among the groups.

When the implant areas prior and subsequent to treatment were compared within each group, a significant decrease in the implant area was found in the leuprolide acetate group. The epithelial MMP-9 immunohistochemical score was additionally significantly lower in the leuprolide acetate group than that in the control group. These findings concerning the leuprolide acetate group in the present study were consistent with those in the study by Akkaya et al (15).

Although the rat endometriosis model proposed by Vernon and Wilson (13), i.e. the autotransplantation of uterine tissues into the peritoneal cavity, is a well-established model, it has certain limitations. Firstly, rats do not menstruate and do not develop spontaneous endometriosis. Secondly, the transplanted uterine tissues include the myometrium, which makes up a considerable proportion of the artificial endometriotic lesions. On the other hand, it has a number of advantages, such as no rejection of the transplanted tissues and enabling long-term studies to study the effects of certain drugs (28). In addition, the pathological findings in the rat model are similar to those in human endometriosis (29).

There are numerous of previous studies investigating the potential therapeutic effects of certain drugs in the rat endometriosis model, which indicated that the drug treatment resulted in significant regression of the endometriotic implants (14-16). However, the studies had methodological differences. The time interval between the laparotomies differed and was reported to be 3 (15), 4 (30) and 6 (16) weeks. Uchiide et al (29) reported that progression of the endometriotic implants declined at 14 days after uterine autotransplantation; therefore, the extended periods between the laparotomies and considerably lower estrogen levels in rats compared with humans may result in spontaneous regression of the endometriotic implants. On the other hand, the time when the pathological and immunohistochemical staining of the endometriotic tissues should be performed is unclear, with previous studies performing them immediately or two months following treatment $(15,16,30)$. Furthermore, the limited number of rats in each group, due to the ethical principles, was another limitation that may weaken the statistical significance of these studies. These problems are also valid for the present study and may be regarded as limitations of the study.

In conclusion, although there was no statistically significant difference, Dox reduced the endometriotic implant area in the rat endometriosis model. Further studies are required to investigate the potential efficacy of Dox in endometriosis due to its widespread use and tolerability.

\section{References}

1. Nezhat FR, Shamshirsaz AA, Yildirim G, Nezhat C and Nezhat C: Pelvic pain, endometriosis, and the role of the gynecologist. In: Pediatric, Adolescent, and Young Adult Gynecology. Altchek A and Deligdisch L (eds.). Wiley-Blackwell, Hoboken, NJ, pp174-194, 2009.

2. Sampson J: Peritoneal endometriosis due to the menstrual dissemination of endothelial tissue into the peritoneal cavity. Am J Obstet Gynecol 14: 422-469, 1927.

3. Osteen KG, Bruner-Tran KL, Keller NR and Eisenberg E: Progesterone-mediated endometrial maturation limits matrix metalloproteinase (MMP) expression in an inflammatory-like environment: a regulatory system altered in endometriosis. Ann NY Acad Sci 955: 37-47; discussion 86-88, 2002.

4. Pitsos M and Kanakas N: The role of matrix metalloproteinases in the pathogenesis of endometriosis. Reprod Sci 16: 717-726, 2009.

5. Wu MH, Shoji Y, Wu MC, Chuang PC, Lin CC, Huang MF and Tsai SJ: Suppression of matrix metalloproteinase-9 by prostaglandin $\mathrm{E}(2)$ in peritoneal macrophage is associated with severity of endometriosis. Am J Pathol 167: 1061-1069, 2005.

6. Chung HW, Wen Y, Chun SH, Nezhat C, Woo BH and Lake Polan M: Matrix metalloproteinase-9 and tissue inhibitor of metalloproteinase-3 mRNA expression in ectopic and eutopic endometrium in women with endometriosis: a rationale for endometriotic invasiveness. Fertil Steril 75: 152-159, 2001.

7. Nap AW, Dunselman GA, de Goeij AF, Evers JL and Groothuis PG: Inhibiting MMP activity prevents the development of endometriosis in the chicken chorioallantoic membrane model. Hum Reprod 19: 2180-2187, 2004.

8. Bruner-Tran KL, Zhang Z, Eisenberg E, Winneker RC and Osteen KG: Down-regulation of endometrial matrix metalloproteinase-3 and -7 expression in vitro and therapeutic regression of experimental endometriosis in vivo by a novel nonsteroidal progesterone receptor agonist, tanaproget. J Clin Endocrinol Metab 91: 1554-1560, 2006

9. Lee CZ, Yao JS, Huang Y, et al: Dose-response effect of tetracyclines on cerebral matrix metalloproteinase-9 after vascular endothelial growth factor hyperstimulation. J Cereb Blood Flow Metab 26: 1157-1164, 2006 
10. Acharya MR, Venitz J, Figg WD and Sparreboom A: Chemically modified tetracyclines as inhibitors of matrix metalloproteinases. Drug Resist Updat 7: 195-208, 2004.

11. Axisa B, Loftus IM, Naylor AR, Goodall S, Jones L, Bell PR and Thompson MM: Prospective, randomized, double-blind trial investigating the effect of doxycycline on matrix metalloproteinase expression within atherosclerotic carotid plaques. Stroke 33: 2858-2864, 2002.

12. Griffin MO, Fricovsky E, Ceballos G and Villarreal F: Tetracyclines: A pleitropic family of compounds with promising therapeutic properties. Review of the literature. Am J Physiol Cell Physiol 299: C539-C548, 2010.

13. Vernon MW and Wilson EA: Studies on the surgical induction of endometriosis in the rat. Fertil Steril 44: 684-694, 1985.

14. Lebovic DI, Kir M and Casey CL: Peroxisome proliferator-activated receptor-gamma induces regression of endometrial explants in a rat model of endometriosis. Fertil Steril 82 Suppl 3: 1008-1013, 2004.

15. Akkaya P, Onalan G, Haberal N, Bayraktar N, Mülayim B and Zeyneloglu HB: Doxycycline causes regression of endometriotic implants: A rat model. Hum Reprod 24: 1900-1908, 2009.

16. Keenan JA, Williams-Boyce PK, Massey PJ, Chen TT, Caudle MR and Bukovsky A: Regression of endometrial explants in a rat model of endometriosis treated with the immune modulators loxoribine and levamisole. Fertil Steril 72: 135-141, 1999.

17. Visse R and Nagase H: Matrix metalloproteinases and tissue inhibitors of metalloproteinases: Structure, function, and biochemistry. Circ Res 92: 827-839, 2003.

18. Collette T, Bellehumeur C, Kats R, Maheux R, Mailloux J, Villeneuve $\mathrm{M}$ and Akoum A: Evidence for an increased release of proteolytic activity by the eutopic endometrial tissue in women with endometriosis and for involvement of matrix metalloproteinase-9. Hum Reprod 19: 1257-1264, 2004.

19. Pan H, Sheng JZ, Tang L, Zhu R, Zhou TH and Huang HF: Increased expression of c-fos protein associated with increased matrix metalloproteinase-9 protein expression in the endometrium of endometriotic patients. Fertil Steril 90: 1000-1007, 2008.

20. Sapadin AN and Fleischmajer R: Tetracyclines: Nonantibiotic properties and their clinical implications. J Am Acad Dermatol 54: 258-265, 2006.
21. Hoyt JC, Ballering J, Numanami H, Hayden JM and Robbins RA: Doxycycline modulates nitric oxide production in murine lung epithelial cells. J Immunol 176: 567-572, 2006.

22. Zhao S, Choksuchat C, Zhao Y, Ballagh SA, Kovalevsky GA and Archer DF: Effects of doxycycline on serum and endometrial levels of MMP-2, MMP-9 and TIMP-1 in women using a levonorgestrel-releasing subcutaneous implant. Contraception 79: 469-478, 2009.

23. Pires PW, Rogers CT, McClain JL, Garver HS, Fink GD and Dorrance AM: Doxycycline, a matrix metalloprotease inhibitor, reduces vascular remodeling and damage after cerebral ischemia in stroke-prone spontaneously hypertensive rats. Am J Physiol Heart Circ Physiol 301: H87-H97, 2011.

24. Björnsson MJ, Havemose-Poulsen A, Stoltze K and Holmstrup P: Influence of the matrix metalloproteinase inhibitor batimastat (BB-94) on periodontal bone destruction in Sprague-Dawley rats. J Periodontal Res 39: 269-274, 2004.

25. Coussens LM, Fingleton B and Matrisian LM: Matrix metalloproteinase inhibitors and cancer: trials and tribulations. Science 295: 2387-2392, 2002.

26. Preshaw PM, Hefti AF, Jepsen S, Etienne D, Walker C and Bradshaw MH: Subantimicrobial dose doxycycline as adjunctive treatment for periodontitis. A review. J Clin Periodontol 31: 697-707, 2004

27. Lamparter S, Slight SH and Weber KT: Doxycycline and tissue repair in rats. J Lab Clin Med 139: 295-302, 2002.

28. Grümmer R: Animal models in endometriosis research. Hum Reprod Update 12: 641-649, 2006.

29. Uchiide I, Ihara T and Sugamata M: Pathological evaluation of the rat endometriosis model. Fertil Steril 78: 782-786, 2002.

30. Oner G, Ozcelik B, Ozgun MT, Serin IS, Ozturk F and Basbug M: The effects of metformin and letrozole on endometriosis and comparison of the two treatment agents in a rat model. Hum Reprod 25: 932-937, 2010. 